First publ. in: Gene 246 (2000), pp. 1-8

\title{
Microsporidia: accumulating molecular evidence that a group of amitochondriate and suspectedly primitive eukaryotes are just curious fungi
}

\author{
Yves Van de Peer ${ }^{\mathrm{a}, *}$, Abdelghani Ben Ali ${ }^{\mathrm{b}}$, Axel Meyer ${ }^{\mathrm{a}}$ \\ ${ }^{a}$ Department of Biology, University of Konstanz, D-78457 Konstanz, Germany \\ ${ }^{\mathrm{b}}$ Department of Biochemistry, University of Antwerp (UIA), Universiteitsplein 1, B-2610 Antwerp, Belgium
}

Received 18 November 1999; received in revised form 18 January 2000; accepted 25 January 2000

Received by W. Martin

\begin{abstract}
Microsporidia are obligate intracellular parasites that have long been considered to be primitive eukaryotes, both on the basis of morphological features and on the basis of molecular, mainly ribosomal RNA-based, phylogenies. However, accumulating sequence data and the use of more sophisticated tree construction methods now seem to suggest that microsporidia share a common origin with fungi and are therefore most probably just curious fungi. In this paper, we describe the current views on the phylogenetic position of the microsporidia and present additional evidence for a close relationship between fungi and microsporidia on the basis of reanalysed ribosomal RNA data. In this respect, the importance of incorporating detailed knowledge of the substitution pattern of sequences into phylogenetic methods is discussed. (C) 2000 Elsevier Science B.V. All rights reserved.
\end{abstract}

Keywords: Among-site rate variation; Large subunit ribosomal RNA; Long-branch attraction

\section{Introduction}

The microsporidia or Microspora (to which belong the genera Vairimorpha, Nosema, Encephalitozoon, Enterocytozoon, and several others) are small, obligate intracellular parasites that parasitize many other eukaryotes. Their infection strategy is unique and highly specialized. As infective spores outside their host, the microsporidia have a thick coat of chitin and protein, while the cytoplasm contains a coiled projectile known as the polar tube. When a spore encounters a host, the polar tube is everted and penetrates the host membrane. Then, the cytoplasm of the spore is injected directly into the host cytoplasm, after which the parasites grow and divide in the host cytoplasm and form new spores until these are released from the host cell into the environment (Canning, 1990). Although the vast majority of infec-

\footnotetext{
* Corresponding author. Tel.: +49-7531-88-2763; fax: +49-7531-88-3018.

E-mail address: yves.vandepeer@uni-konstanz.de (Y. Van de Peer)
}

tions by microsporidia have been described in metazoans (e.g. Didier et al., 1998; Moser et al., 1998; Nilsen et al., 1998; Nilsen, 1999), some have also been characterized in protists such as ciliates and Apicomplexans (e.g. Dykova and Lom, 1999). In humans, infections are mostly associated with patients that have compromised immune systems, such as HIV-infected patients (e.g. Canning and Hollister, 1992; Dascomb et al., 1999).

Until quite recently, microsporidians were believed to be the descendants of a primitive, ancient eukaryotic lineage. Besides missing typical eukaryotic features such as peroxisomes, classical stacked Golgi membranes, and $9+2$ microtubule structures, the absence of mitochondria in particular suggested an ancient origin of these organisms. This even resulted in classifying these organisms as Archezoa or 'old' eukaryotes (CavalierSmith, 1989; Roger, 1999), together with several other amitochondriate, enigmatic eukaryotic groups such as the diplomonadida (e.g. Giardia), and parabasalids (e.g. Trichomonas), which were all thought to have originated before the event that led to the mitochondrion endosymbiosis. 
In 1987, the first small subunit ribosomal RNA (SSU rRNA) sequence of a microsporidian (Vairimorpha necatrix) was determined (Vossbrinck et al., 1987), being the first molecular marker that became available to study the evolutionary relationships for this group of organisms. Since, in phylogenetic trees, this SSU rRNA sequence branched off before any other eukaryotic SSU rRNA sequence known at that time, the early origin and uniqueness of these parasites within eukaryotic evolution seemed confirmed. Subsequent analyses, on the basis of the SSU rRNA of Vairimorpha and other microsporidia, and using different methods of tree construction (Sogin, 1989; Van de Peer et al., 1993a; Cavalier-Smith and Chao, 1996; Kumar and Rzhetsky, 1996), all supported the view that microsporidia were an early-diverging and therefore presumably extremely old lineage from which the ancestors originated long before the remaining eukaryotes, amongst which are the fungi, plants, animals and many others that form the so-called crown group of eukaryotic evolution (Knoll, 1992). This 'microsporidia-early' (Hirt et al., 1999) hypothesis was also supported by recent phylogenetic analyses of the protein translation elongation factor sequences (EF-1 $\alpha$ and EF-2; Kamaishi et al., 1996a,b).

\section{Primitive or highly degenerated?}

However, this view about 'old' microsporidian origins has recently been challenged. Firstly, it was shown that microsporidians, but also the other amitochondrial eukaryotes mentioned above, probably once harbored mitochondria since mitochondrial genes have been found in their nuclear genomes (Clark and Roger, 1995; Germot et al., 1997; Hirt et al., 1997; Hashimoto et al., 1998; Peyretaillade et al., 1998a; Roger et al., 1998), thus pointing to a secondary amitochondriate nature for these organisms. Secondly, regarding the evolution of microsporidia, recent phylogenetic studies on the basis of protein-coding genes such as tubulin, the largest subunit of the RNA polymerase II (RPB1), valyl-tRNA synthetase (ValRS), and the TATA box binding protein (TBP) showed a close relationship of these parasitic organisms with the fungi (Edlind et al., 1996; Keeling and Doolittle, 1996; Fast et al., 1999; Hirt et al., 1999; Weiss et al., 1999). Furthermore, although phylogenetic analysis of the elongation factor data usually indicates an early origin of microsporidia (Kamaishi et al., 1996a,b; but see further), the EF-1 $\alpha$ gene from the microsporidian Glugea plecoglossi shares a unique insertion of 11 amino acids with animals and fungi (Kamaishi et al., 1996a). Hence, if such a close relationship between fungi and microsporidia were indeed real, then this implies that their simplified cell structures and small genomes (Biderre et al., 1995) are secondary simplified and degenerate features, and probably the result of their highly specialised parasitic lifestyles.

The apparent discrepancy between phylogenetic hypotheses based on protein coding genes and trees based on ribosomal rRNA sequences has been troublesome for some time and still needs to be fully understood. However, it was repeatedly suggested that the early divergence patterns of some protist phyla, and in particular the microsporidia, are long-branch artefacts due to increased evolutionary rates of their ribosomal RNAs (e.g. Palmer and Delwiche, 1996; Embley and Hirt, 1998; Keeling and McFadden, 1998).

\section{Among-site rate variation and ribosomal RNA revisited}

Long-branch artefacts have gained much interest in the last few years, and are now generally considered to be a major source of distorted tree topologies (e.g. Forterre and Philippe, 1999). This is especially true when there is considerable among-site rate variation in the sequences considered (e.g. Van de Peer et al., 1996a). The existence of among-site rate variation implies that the majority of mutations take place at the same, relatively small fraction of positions. Naturally, this is even more pronounced when distances between sequences are large, as is the case in rapidly evolving sequences, i.e. sequences characterized by forming long branches in the inferred tree topologies. Consequently, genetic distances are seriously underestimated if site-tosite rate variations are not taken into account. This often results in an artificial clustering of long branches, or in long branches being pulled closer to the base of the tree in the presence of a distantly related outgroup (Felsenstein, 1978; Olsen, 1987; Yang, 1996; Brinkmann and Philippe, 1999).

In the present study, we have aligned the recently published large subunit (LSU) ribosomal RNA sequences of two microsporidia, viz. Encephalitozoon cuniculi (Peyretaillade et al., 1998b) and Nosema apis (Gatehouse and Malone, 1998) with those of the other eukaryotes for which the LSU rRNA is available (De Rijk et al., 2000). Additionally, we determined the LSU rRNA sequences of two ciliates, viz. Spathidium amphoriforme (Accession No. AF223570) and Euplotes aediculatus (Accession No. AF223571). The LSU rRNA sequences of microsporidia are the shortest non-mitochondrial LSU rRNAs known so far (De Rijk et al., 1998; Peyretaillade et al., 1998a). The strong reduction in size can be attributed to changes in the regions that are known to show a large variability in length and sequence. In these areas, eukaryotes can exhibit extreme variations in sequence and/or length in comparison to prokaryotes and other eukaryotes. However, despite the seemingly prokaryote-like features of the LSU rRNA, 
such as the strongly reduced size and absence of fragmentation as in other eukarotes, they cannot be used as evidence for the ancient origin of the microsporidia. Indeed, although the 5.8S rRNA typically found in eukaryotes is fused with the rest of the LSU rRNA the microsporidia are the only eukaryotes known to date to show such a fusion - the structure of the fusion is different from that seen in prokaryotes and other eukaryotes. Furthermore, the regions responsible for the reduction in size are known to show a large variation within eukaryotes, and in the microsporidia, these areas show no obvious similarity to their prokaryotic counterparts either.

Despite the strong reduction in length of the microsporidian LSU rRNAs, a major part of the sequence can be aligned to other eukaryotic sequences, and unambiguously aligned regions were subjected to phylogenetic analyses based on the 'substitution rate calibration' (SRC) method, which corrects for site-to-site rate variation in nucleic acid sequences (Van de Peer et al., 1996b). An estimation of substitution rates and construction of a variability map was performed as described previously (Van de Peer et al., 1993b, 1996b,c). In short, substitution rates are estimated by looking at the frequency with which sequence pairs differ at each site as a function of the distance between them. Substitution rates or variabilities are estimated for every site in the sequence alignment that is not absolutely conserved and that contains a nucleotide in at least $25 \%$ of the aligned sequences. Then, after estimation of all substitution rates, alignment positions are grouped into sets of similar rates. A spectrum of relative nucleotide substitution rates is thus obtained. By dividing this spectrum into five arbitrary groups and assigning a different colour to every subset, a variability chart of the LSU rRNA can be constructed by mapping the five variability classes on the secondary structure model of the LSU rRNA. Such a variability map, which gives a much more detailed and quantitative description of positional variability than the crude distinction between variable and conserved areas that is often made by visual inspection of sequence alignments, is shown for Nosema apis (De Rijk et al., 1998) in Fig. 1. Furthermore, once the shape of the rate spectrum is known, it is possible to derive a new equation describing the evolutionary distance between two sequences as a function of the observed number of differences, i.e. the dissimilarity (Van de Peer et al., 1996b). This new equation, similar to that used to compute gamma distances (Rzhetsky and Nei, 1994), can then be used to compute evolutionary distances, taking into account the specific substitution rate spectrum for the LSU rRNA sequence alignment considered. These evolutionary distances can then be used for the inference of a distance tree.

Fig. 2a shows a phylogenetic tree based on 42 sequences of representatives of the different eukaryotic crown taxa plus the sequences of the microsporidia Nosema and Encephalitozoon. The tree shown was artificially rooted between the green plants and the remaining eukaryotic taxa. Trees that were rooted with Giardia or Archaea (not shown) basically showed the same topology, although in some cases, the plants appeared as a paraphyletic group near the base of the tree. As can be seen in Fig. 2a, the microsporidia diverge from within the fungal cluster, although bootstrap support for a common origin of microsporidia and fungi is low (62\%). However, this may not be very surprising since the branch leading to the microsporidia is exceptionally long (Fig. 2a). Also, in rate-calibrated trees based on smaller data sets, microsporidia nearly always diverge from within the fungal cluster, although usually not strongly statistically supported by bootstrap analysis, while the overall tree topology corresponds well with what we would expect on the basis of more classical phylogenetic analyses from small and large subunit rRNA data (e.g. Van de Peer and De Wachter, 1997a). When the among-site rate variation is not considered, the microsporidia branch off towards the base of the tree and do not cluster with any of the other taxa. A similar phenomenon was also previously observed by Peyretaillade et al. (1998b), where the microsporidian Encephalitozoon diverged early in eukaryote evolution when different substitution rates were not taken into account, but relatively late when different rates were considered. However, a close relationship with fungi could not be demonstrated (Peyretaillade et al., 1998b).

In order to test whether better tree topologies could be found on a maximum likelihood basis, we performed a Kishino-Hasegawa test (Kishino and Hasegawa, 1989), where we compared the maximum likelihood of the SRC tree of Fig. 2a, with that of different userdefined trees, using the same data set - with invariant positions removed, as in the SRC method - and rate parameter as estimated by the SRC method. Conclusively, trees where the microsporidia branched off at the base of the fungal, animal, and fungal-animal cluster were not found to be significantly better or worse than the tree presented in the current paper (Fig. 2a). However, user-defined trees where microsporidia clustered with other taxa, or branched off more deeply, were found to be significantly worse in the Kishino-Hasegawa tests (as implemented in PAUP, version 4, Swofford, 1998), with the exception of a tree topology in which microsporidia were clustered with ciliates, for which the likelihood was not significantly worse. However, we believe that the better likelihood of this tree is the result of artificially grouping organisms with higher evolutionary rates; the ciliates indeed show a somewhat higher evolutionary rate of their rRNAs compared to most other eukaryotes in the tree.

Additionally, a maximum likelihood tree was com- 


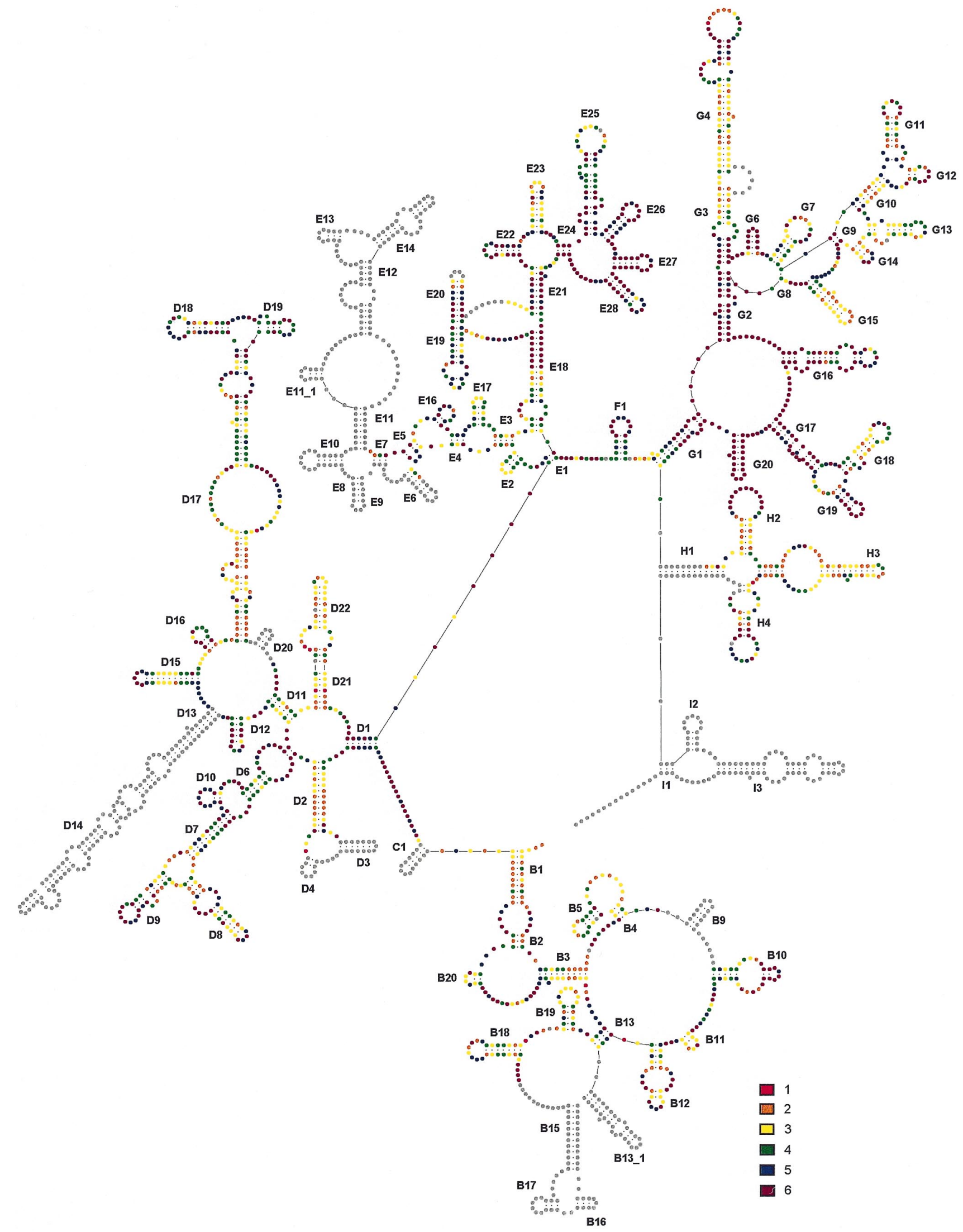

Fig. 1. Variability map superimposed on the LSU rRNA secondary structure model of Nosema apis (De Rijk et al., 1998). Nucleotides are subdivided into 5 groups of increasing variability as described in Ben Ali et al. (1999). The most variable positions are in red, and the least variable positions in blue. Absolutely conserved positions in all sequences compared are indicated in purple. Hypervariable regions that were not taken into consideration for rate calibration and tree construction because they were too variable to be aligned unambiguously are indicated in grey. These are also the regions that are responsible for the great reduction in size of the microsporidian LSU rRNAs. 


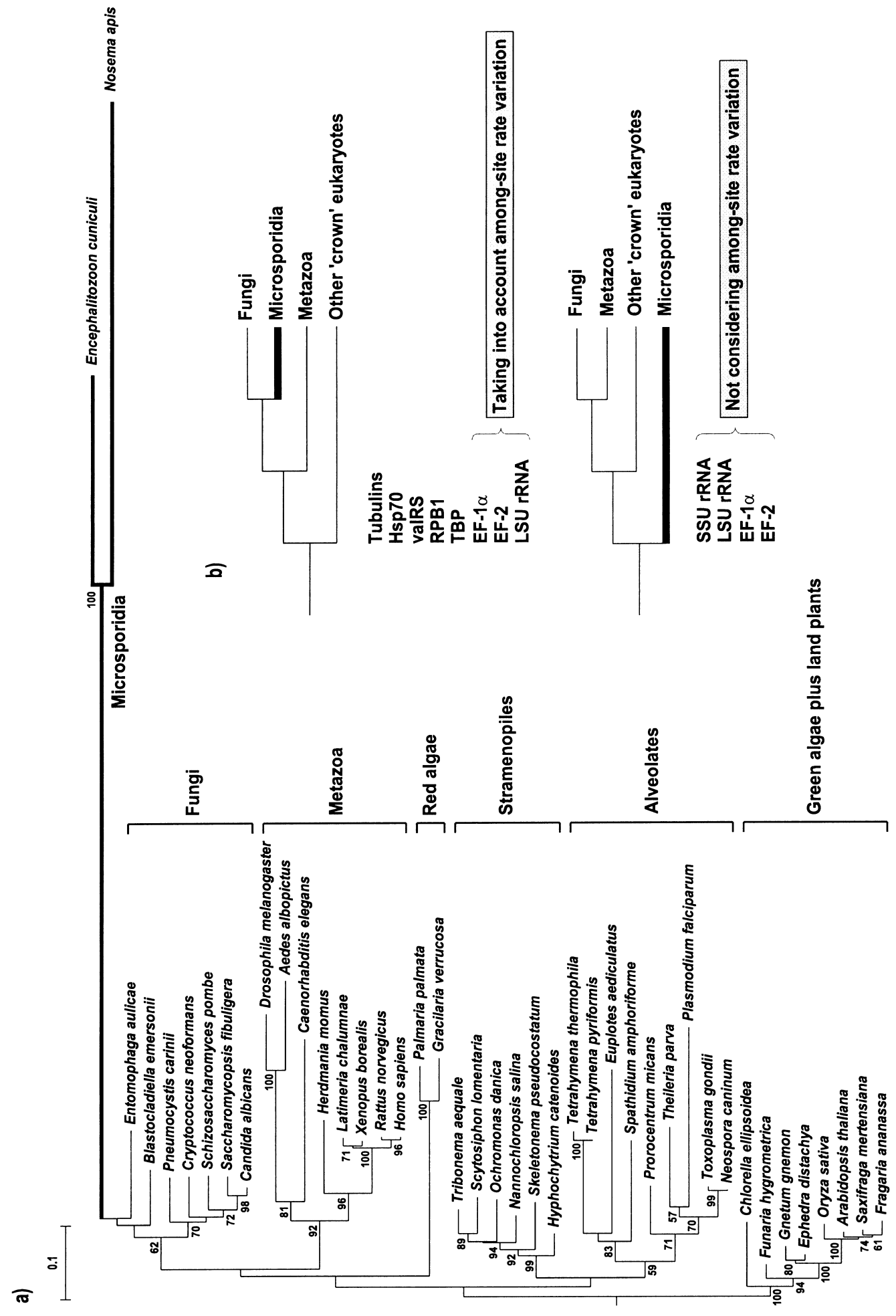

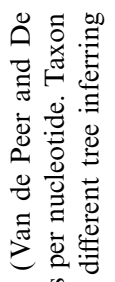

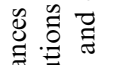

营

可 券

을

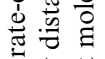

记

웅

要

के

을

氜

过

语

है छ

过

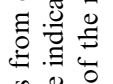

过

可

记

Z

는

需言

它这离

岸

过

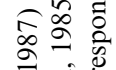

$\Rightarrow$ घ

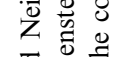

空

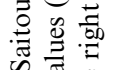

bo 㐘

등 훙

車它

क्षे

乙。े

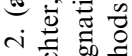

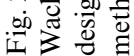


puted with the Puzzle program (Strimmer and von Haeseler, 1996), based on the same set of positions (see Fig. 1) used to compute the SRC distance tree (Fig. 2a). The among-site rate variation was taken into account by assuming eight different rate categories, while the gamma distribution parameter was estimated from the data set. This tree (not shown) showed almost exactly the same topology as the SRC tree, while the quartet puzzling values - comparable, but not equal, to bootstrap values (Strimmer and von Haeseler, 1996) - were also very similar to the bootstrap values found for the tree shown in Fig. 2a. As in the SRC tree, the microsporidia were clustered with the zygomycete, Entomophaga, and the chytridiomycete, Blastocladiella, but this relationship should be interpreted with caution and is not at all supported by bootstrap analysis (Fig. 2a) or quartet puzzling values (not shown). In the maximum likelihood tree, animals and fungi are also found as sister groups, as are the alveolates and the stramenopiles, although neither of the sister group relationships is statistically supported.

Fig. $2 \mathrm{~b}$ summarises the two topologies most frequently found regarding the evolutionary position of the microsporidia and their support by different molecular markers. As already stated, tubulins, Hsp70, RPB1, ValRS, and TBP supported the 'microsporidia-late' hypothesis, while SSU rRNA, LSU rRNA and elongator factor genes initially supported the 'microsporidia-early' hypothesis. However, when the among-site rate variation is taken into account, the picture changes, as already described in detail above for the LSU rRNA. Regarding the elongation factor data sets, Hirt et al. (1999) re-examined EF- $1 \alpha$ and EF-2 and found that these data sets were not inconsistent with a fungi-plus-microsporidia relationship, as long as the among-site rate variation was considered or at least highly reduced (Hirt et al., 1999). Applying the SRC method to SSU rRNA sequences caused the microsporidia to diverge from within the eukaryotic crown, although a specific relationship with fungi cannot currently be firmly established.

\section{Conclusions}

To our knowledge, this paper shows, for the first time, relatively strong evidence for a common ancestry of fungi and microsporidia on the basis of ribosomal RNA data, although unequivocal statistical support is still lacking. However, this should not be very surprising regarding the complexity of the problem and the amazing length of the branch leading to the microsporidia. Therefore, together with recent phylogenies constructed on the basis of protein data, and similarities such as the presence of chitin and trehalose and similar characteristics of the meiotic and mitotic cycles (Sprague et al., 1992; Keeling and Doolittle, 1996; Germot et al., 1997), evidence for a close evolutionary relationship between fungi and microsporidia has now become quite convincing.

The microsporidia problem described here again exemplifies one of the complexities of inferring reliable tree topologies. The difference in tree topology supporting a microsporidia-early or microsporidia-late origin is huge, yet good statistical support can be found for both topologies (e.g. Cavalier-Smith and Chao, 1996; Keeling and Doolittle, 1996; Hirt et al., 1999). Nevertheless, consistency in tree topologies can be reached, provided adequate tree-building methods are used. In order to do this, it is crucial to use substitution models that describe the evolution of the sequences as realistically and accurately as possible. However, since we do not have an exact historical record of events that took place in the evolution of our sequences, a correct estimation of the number of substitutions is usually not straightforward. Fortunately, more realistic evolution (substitution) models and new and better methods for inferring phylogenetic tree topologies are continuously being developed, which hopefully will lead to more consistent tree topologies as is the case with the microsporidia.

Apart from drastically changing our view about early eukaryotic evolution - the microsporidia example sheds doubt on the phylogenetic position of other early branching protists as well (e.g. Embley and Hirt, 1998; Keeling, 1998) — solving the microsporidia enigma may have important medical consequences too. Microsporidia are important parasites causing, amongst other things, diarrhoea and weight loss in AIDS patients, and a completely effective treatment has not yet been discovered. However, if microsporidia are indeed fungi this could have important implications for future treatment, as was previously the case with Pneumocystis carinii, whose taxonomy was uncertain until molecular data undoubtedly proved it to be a yeast (Edman et al., 1988), after which treatment of AIDS patients became much more efficient.

\section{Acknowledgements}

Y.V.d.P. is Research Fellow of the National Fund for Scientific Research (Belgium) and acknowledges financial support from the University of Konstanz (Germany) and the University of Antwerp (Belgium). Support from the Fond der Chemischen Industrie and the DFG to A.M. is also acknowledged. We want to thank Andre Adoutte for DNA of ciliates and Henner Brinkmann for help with phylogenetic analyses and critical comments.

\section{References}

Ben Ali, A., Wuyts, J., De Wachter, R., Meyer, A., Van de Peer, Y., 1999. Construction of a variability map for eukaryotic large subunit ribosomal RNA. Nucleic Acids Res. 27, 2825-2831. 
Biderre, C., Pages, M., Méténier, G., Canning, E.U., Vivarès, C.P., 1995. Evidence for the smallest nuclear genome $(2.9 \mathrm{Mb})$ in the microsporidium Encephalitozoon cuniculi. Mol. Biochem. Parasitol. 74, 229-231.

Brinkmann, H., Philippe, H., 1999. Archaea sister group of bacteria? Indications from tree reconstruction artifacts in ancient phylogenies. Mol. Biol. Evol. 16, 817-825.

Canning, E.U., 1990. Phylum Microsporidia. In: Margulis, L., Corliss, J.O., Melkonian, M., Chapman, D.J. (Eds.), Handbook of Protoctista. Jones and Bartlett, Boston, MA, pp. 53-72.

Canning, E.U., Hollister, W.S., 1992. Human infections with microsporidia. Rev. Med. Microbiol. 3, 35-42.

Cavalier-Smith, T., 1989. Archaebacteria and Archezoa. Nature 339, $100-101$.

Cavalier-Smith, T., Chao, E.E., 1996. Molecular phylogeny of the freeliving Archezoan Trepomonas agilis and the nature of the first eukaryote. J. Mol. Evol. 43, 551-562.

Clark, C.G., Roger, A.J., 1995. Direct evidence for secondary loss of mitochondria in Entamoeba histolytica. Proc. Natl. Acad. Sci. USA 92, 6518-6521.

Dascomb, K., Clark, R., Aberg, J., Pulvirenti, J., Hewitt, R.G., Kissinger, P., Didier, E.S., 1999. Natural history of intestinal microsporidiosis among patients infected with human immunodeficiency virus. J. Clin. Microbiol. 37, 3421-3422.

De Rijk, P., Gatehouse, H.S., De Wachter, R., 1998. The secondary structure of Nosema apis large subunit ribosomal RNA. Biochim. Biophys. Acta 1442, 326-328.

De Rijk, P., Wuyts, J., Van de Peer, Y., Winkelmans, T., De Wachter, R., 2000. The European large subunit ribosomal RNA database. Nucleic Acids Res. 28, 177-178.

Didier, E.S., Snowden, K.F., Shadduck, J.A., 1998. Biology of microsporidian species infecting mammals. Adv. Parasitol. 40, 283-320.

Dykova, I., Lom, J., 1999. Nosema notabilis (Microsporidia), its ultrastructure and effect on the myxosporean host Ortholinea polymorpha. Dis. Aquat. Organ. 35, 69-76.

Edlind, T.D., Li, J., Visvesvara, G.S., Vodkin, M.H., McLaughlin, G.L., Katiyar, S.K., 1996. Phylogenetic analysis of $\beta$-tubulin sequences from amitochondrial Protozoa. Mol. Phyl. Evol. 5, 359-367.

Edman, J.C., Kovacs, J.A., Masur, H., Santi, D.V., Elwood, H.J., Sogin, M.L., 1988. Ribosomal RNA sequence shows Pneumocystis carinii to be a member of the fungi. Nature 334, 519-522.

Embley, T.M., Hirt, R.P., 1998. Early branching eukaryotes. Curr. Opin. Genet. Dev. 8, 624-629.

Fast, N.M., Logsdon Jr., J.M., Doolittle, W.F., 1999. Phylogenetic analysis of the TATA box binding protein (TBP) gene from Nosema locustae: evidence for a microsporidia-fungi relationship and spliceosomal intron loss. Mol. Biol. Evol. 16, 1415-1419.

Felsenstein, J., 1978. Cases in which parsimony and compatibility methods will be positively misleading. Syst. Zool. 27, 401-410.

Felsenstein, J., 1985. Confidence limits on phylogenies: an approach using the bootstrap. Evolution 39, 783-791.

Forterre, P., Philippe, H., 1999. Where is the root of the universal tree of life? BioEssays 21, 871-879.

Gatehouse, H.S., Malone, A., 1998. The ribosomal RNA gene region of Nosema apis (Microspora): DNA sequence for small and large subunit rRNA genes and evidence of a large tandem repeat unit size. J. Invertebr. Pathol. 71, 97-105.

Germot, A., Philippe, H., Le Guyader, H., 1997. Evidence for loss of mitochondria in Microsporidia from a mitochondrial-type HSP70 in Nosema locustae. Mol. Biochem. Parasitol. 87, 159-168.

Hashimoto, T., Sanchez, L.B., Shirakura, T., Muller, M., Hasegawa, M., 1998. Secondary absence of mitochondria in Giardia lamblia and Trichomonas vaginalis revealed by valyl-tRNA synthetase phylogeny. Proc. Natl. Acad. Sci. USA 95, 6860-6865.

Hirt, R.P., Healy, B., Vossbrinck, C.R., Canning, E.U., Embley, T.M., 1997. A mitochondrial Hsp70 orthologue in Vairimorpha necatrix: molecular evidence that microsporidia once contained mitochondria. Curr. Biol. 7, 995-998.

Hirt, R.P., Logsdon Jr., J.M., Healey, B., Dorey, M.W., Doolittle, W.F., Embley, T.M., 1999. Microsporidia are related to Fungi: evidence from the largest subunit of RNA polymerase II and other proteins. Proc. Natl. Acad. Sci. USA 96, 580-585.

Kamaishi, T., Hashimoto, T., Nakamura, Y., Masuda, Y., Nakamura, F., Okamoto, K., Shimizu, M., Hasegawa, M., 1996a. Complete nucleotide sequences of the genes encoding translation elongation factors $1 \alpha$ and 2 from a microsporidian parasite, Glugea plecoglossi: implications for the deepest branching of eukaryotes. J. Biochem. 120, 1095-1103.

Kamaishi, T., Hashimoto, T., Nakamura, Y., Nakamura, F., Murata, S., Okada, N., Okamoto, K., Shimizu, M., Hasegawa, M., 1996b. Protein phylogeny of traslation elongation factor Ef- $1 \alpha$ suggests microsporidians are extremely ancient eukaryotes. J. Mol. Evol. 42, 257-263.

Keeling, P.J., Doolittle, W.F., 1996. Alpha-tubulin from early-diverging eukaryotic lineages and the evolution of the tubulin family. Mol. Biol. Evol. 13, 1297-1305.

Keeling, P.J., 1998. A kingdom's progress: archezoa and the origin of eukaryotes. BioEssays 20, 87-95.

Keeling, P.J., McFadden, G.I., 1998. Origins of microsporidia. Trends Microbiol. 19, 19-23.

Kishino, H., Hasegawa, M., 1989. Evaluation of the maximum likelihood estimate of the evolutionary tree topologies from DNA sequence data, and the branching order in hominoidea. J. Mol. Evol. 29, 170-179.

Knoll, A.H., 1992. The early evolution of eukaryotes: a geological perspective. Science 256, 622-627.

Kumar, S., Rzhetsky, A., 1996. Evolutionary relationships of eukaryotic kingdoms. J. Mol. Evol. 42, 183-193.

Moser, B.A., Becnel, J.J., Maruniak, J., Patterson, R.S., 1998. Analysis of the ribosomal DNA sequences of the microsporidia Thelohania and Vairimorpha of fire ants. J. Invertebr. Pathol. 72, 154-159.

Nilsen, F., Endresen, C., Hordvik, I., 1998. Molecular phylogeny of microsporidians with particular reference to species that infect the muscles of fish. J. Eukaryot. Microbiol. 45, 535-543.

Nilsen, F., 1999. Small subunit rDNA phylogeny of Bacillidium sp. (Microspora, Mrazekiidae) infecting oligochaets. Parasitology $118,553-558$.

Olsen, G.J., 1987. Earliest phylogenetic branchings: comparing rRNAbased evolutionary trees inferred with various techniques, Cold Spring Harbor Symposia on Quantitative Biology LII, 825-837.

Palmer, J.D., Delwiche, C.F., 1996. Second-hand chloroplasts and the case of the disappearing nucleus. Proc. Natl. Acad. Sci. USA 93, $7432-7435$.

Peyretaillade, E., Broussolle, V., Peyret, P., Méténier, G., Gouy, M., Vivarès, C.-P., 1998a. Microsporidia, amitochondrial protists, posses a $70-\mathrm{kDa}$ heat shock protein gene of mitochondrial evolutionary origin. Mol. Biol. Evol. 15, 683-689.

Peyretaillade, E., Biderre, C., Peyret, P., Duffieux, F., Méténier, G., Gouy, M., Michot, B., Vivarès, C.P., 1998b. Microsporidian Encephalitozoon cuniculi, a unicellular eukaryote with an unusual chromosomal dispersion of ribosomal genes and a LSU rRNA reduced to the universal core. Nucleic Acids Res. 26, 3513-3520.

Rzhetsky, A., Nei, M., 1994. Unbiased estimates of the number of nucleotide substitutions when substitution rate varies among different sites. J. Mol. Evol. 38, 295-299.

Roger, A.J., Svard, S.G., Tovar, J., Clark, C.G., Smith, M.W., Gillin, F.D., Sogin, M.L., 1998. A mitochondrial-like chaperonin 60 gene in Giardia lamblia: evidence that diplomonads once harbored an endosymbiont related to the progenitor of mitochondria. Proc. Natl. Acad. Sci. USA 95, 229-234.

Roger, A.J., 1999. Reconstructing early events in eukaryotic evolution. Am. Nat. 154, S146-S163. 
Saitou, N., Nei, M., 1987. The neighbor-joining method: a new method for reconstructing phylogenetic trees. Mol. Biol. Evol. 4, 406-425.

Sogin, M.L., 1989. Evolution of eukaryotic microorganisms and their small subunit ribosomal RNAs. Am. Zool. 29, 487-499.

Sprague, V., Becnel, J.J., Hazard, E.I., 1992. Taxonomy of phylum Microspora. Crit. Rev. Microbiol. 18, 285-395.

Strimmer, K., von Haeseler, A., 1996. Quartet puzzling: a quartet maximum likelihood method for reconstructing tree topologies. Mol. Biol. Evol. 13, 964-969.

Swofford, D.L., 1998. PAUP* Phylogenetic Analysis Using Parsimony ( ${ }^{*}$ and Other Methods) Version 4. Sinauer Associates, Sunderland, MA.

Van de Peer, Y., Neefs, J.M., De Rijk, P., De Wachter, R., 1993a. Evolution of eukaryotes as deduced from small ribosomal subunit RNA sequences. Biochem. Syst. Ecol. 21, 43-55.

Van de Peer, Y., Neefs, J.M., De Rijk, P., De Wachter, R., 1993 b. Reconstructing evolution from eukaryotic small ribosomal subunit RNA sequence: Calibration of the molecular clock. J. Mol. Evol. 37, 221-232.

Van de Peer, Y., Rensing, S., Maier, U.G., De Wachter, R., 1996a. Substitution rate calibration of small ribosomal subunit RNA identifies chlorarachniophyte endosymbionts as remnants of green algae. Proc. Natl. Acad. Sci. USA 93, 7732-7736.
Van de Peer, Y., Van der Auwera, G., De Wachter, R., 1996b. The evolution of stramenopiles and alveolates as derived by 'substitution rate calibration' of small ribosomal subunit RNA. J. Mol. Evol. 42, 201-210.

Van de Peer, Y., Chapelle, S., De Wachter, R., 1996c. A quantitative map of nucleotide substitution rates in bacterial ribosomal subunit RNA. Nucleic Acids Res. 24, 3381-3391.

Van de Peer, Y., De Wachter, R., 1997a. Evolutionary relationships among the eukaryotic crown taxa taking into account site to site rate variation in 18S rRNA. J. Mol. Evol. 45, 619-630.

Van de Peer, Y., De Wachter, R., 1997b. Construction of evolutionary distance trees with TREECON for Windows: accounting for variation in nucleotide substitution rate among sites. Comput. Appl. Biosci. 13, 227-230.

Vossbrinck, C.R., Maddox, J.V., Friedman, S., Debrunner-Vossbrinck, B.A., Woese, C.R., 1987. Ribosomal RNA sequence suggests microsporidia are extremely ancient eukaryotes. Nature 326, 411-414.

Weiss, L.M., Edlind, T.D., Vossbrinck, C.R., Hashimoto, T., 1999. Microsporidian molecular phylogeny: the fungal conncetion. J. Eukaryot. Microbiol. 46, 17S-18S.

Yang, Z., 1996. Among-site rate variation and its impact on phylogenetic analyses. Trends Ecol. Evol. 11, 367-372. 